\title{
Anti-allergic effect of luteolin in mice with allergic asthma and rhinitis
}

\author{
TAE YOUNG JANG, AH-YEOUN JUNG, TAE-SUK KYUNG, DAE-YOUNG KIM, JUN-HA HWANG, \\ YOUNG HYO KIM
}

Department of Otorhinolaryngology, Head and Neck Surgery, Inha University College of Medicine, Incheon, Republic of Korea

\begin{abstract}
Aim of the study: We aimed to evaluate the anti-allergic effect of luteolin treatment in mice with allergic asthma and rhinitis.

Material and methods: Thirty-two BALB/c mice ( $n=8$ for each group) were used. Mice in group $A$ (nonallergic group) were exposed to saline, while those in Group B (allergic group) were exposed to ovalbumin (OVA) intraperitoneal (i.p.) injection and intranasal (i.n.) challenge. Null treatment group $($ Group $C)$ received sterile saline $(150 \mu l)$ i.p. injection, 30 minutes before each i.n. challenge. Finally, the treatment group (Group D) received luteolin $(0.1 \mathrm{mg} / \mathrm{kg}$ ) by i.p. injection, 30 minutes before each i.n. challenge. We evaluated the number of inflammatory cells including eosinophils, neutrophils and lymphocytes in bronchoalveolar lavage (BAL) fluid, the titers of IL-4, IL-5 and IL-13 in lung homogenate, and we also evaluated histopathologic findings, including infiltration of inflammatory cells into the pulmonary parenchyma and nasal mucosa.

Results: After the OVA challenge, the number of eosinophils, neutrophils and lymphocytes in BAL fluid was significantly increased in group $B$, compared to group $A(p<0.001)$. Mice in group $C$ had no significant difference $(p>0.05)$. On the other hand, group $D$ showed a significant decrease in all inflammatory cells compared to group $B(p<0.05)$. Also, group D showed a significant decrease in $I L-4, I L-5$ and $I L-13$ in their lung homogenate compared to groups $B$ and $C(p<0.05)$. Group D also showed a significant decrease in inflammatory cell infiltration after luteolin treatment $(p<0.05)$.

Conclusion: Luteolin had an anti-allergic effect in a murine model of allergic asthma and rhinitis.
\end{abstract}

Key words: asthma, allergic rhinitis, luteolin.

(Cent Eur J Immunol 2017; 42 (1): 24-29)

\section{Introduction}

Allergic asthma and rhinitis are inflammatory diseases of the upper and lower airway, characterized by induction of Th2 inflammatory response. They are very common diseases and have a negative effect on the life quality of patients. The current medications include bronchodilators such as $\beta 2$ agonists, inhaled and systemic corticosteroids, antihistamines and leukotriene modifiers, all only with transient symptomatic relief.

Luteolin is a kind of a plant-derived natural compound with an immunomodulatory effect [1]. According to its anti-inflammatory effects, it is possible that luteolin could be a useful therapeutic agent against allergic asthma and rhinitis. Although some researchers had suggested that luteolin could reduce bronchoconstriction and airway hyperresponsiveness in allergic mice [1], there is still no study about the effect of luteolin on the infiltration of inflammatory cells into the pulmonary parenchyma and nasal cavity.
Therefore, we evaluated the anti-allergic effect of luteolin by measuring: (a) the number of inflammatory cells such as eosinophils, neutrophils and lymphocytes in bronchoalveolar lavage (BAL) fluid, (b) the titer of Th2 cytokines such as IL-4, IL-5 and IL-13 in pulmonary parenchyma and (c) the degree of infiltration of inflammatory cells into the lung parenchyma and nasal mucosa.

\section{Material and methods}

\section{Animals}

We used 32 female BALB/c mice, 6 to 8 weeks old and free from any murine-specific pathogens (Orient Bio, Seongnam, Korea). They were kept in a well-controlled environment with a regular 12-hour light-dark cycle and unrestricted access to ovalbumin (OVA)-free food and water. All mice used for this study were handled according to a protocol approved by the Animal Care and Use Committee of Inha University (INHA-150309-351). All of our

Correspondence: Young Hyo Kim, MD, PhD, Department of Otorhinolaryngology, Head and Neck Surgery, Inha University College of Medicine, Inhang-ro 27, Jung-Gu, Incheon 22332, Republic of Korea, e-mail: inhaorl@inha.ac.kr, tel. 82-32-890-2437, fax: 82-32-890-3580 Submitted: 13.05.2016, Accepted: 24.06.2016. 
experiments were performed in accordance with approved guidelines and regulations.

\section{Systemic sensitization and intranasal challenge}

To induce allergic asthma and rhinitis, we adopted a published protocol with slight modifications [2-4]. Under pathogen-free conditions, $25 \mu \mathrm{g}$ OVA (Sigma-Aldrich, St. Louis, MO) diluted in sterile saline and aluminum hydroxide gel (alum adjuvant, $2 \mathrm{mg}$ ) was injected intraperitoneally (i.p.) in a total volume of $150 \mu \mathrm{l}$ on experimental days 1 , 5,14 , and 21 for systemic sensitization. Thereafter, daily intranasal instillation (i.n.) of OVA diluted in sterile saline (20 $\mu \mathrm{l}$ of $25-\mathrm{mg} / \mathrm{ml}$ OVA per mouse) was performed until experimental day 35 (14 challenges in total).

The mice were divided into four groups $(n=8 \mathrm{mice} /$ group). Negative control animals (group A, nonallergic group) were exposed to sterile saline, while positive control animals (Group B, allergic group) were exposed to OVA i.p. injection and i.n. challenge. Null treatment group (Group C) received sterile saline $(150 \mu \mathrm{l})$ by i.p. injection, 30 minutes before each i.n. challenge. Finally, the treatment group (Group D) received luteolin $(0.1 \mathrm{mg} / \mathrm{kg})$ by i.p. injection, 30 minutes before each i.n. challenge.

\section{Serum and BAL fluid harvest}

Immediately after sacrifice, we cannulated the trachea using polyethylene tubing and then used a pulmonary lavage technique with sterile saline to harvest BAL fluid (approximately $3 \mathrm{ml}$ ). To determine the viability of cells and the total cell count, we used the trypan blue exclusion assay. Total cell numbers were determined in duplicates with a hemocytometer. Subsequently, a 100- to 200- $\mu$ l aliquot was centrifuged in a Model 2 Cytospin cytocentrifuge (Shandon Scientific, Pittsburgh, PA, USA). Differential cell counts for eosinophils, neutrophils, and lymphocytes were determined from centrifuged preparations stained with the Diff-Quik stain kit (Sysmex Corp., Kobe, Japan) by counting 500 or more cells from each sample at a magnification of $200 \times$ (oil immersion).

\section{Enzyme-linked immunosorbent assay (ELISA)}

We evaluated the levels of cytokines by ELISA as described previously, using the lung homogenate tissue [5]. The titers of IL-4, IL-5, and IL-13 were measured using individual ELISA kits (BD Biosciences, San Diego, CA, USA) according to the manufacturer's instructions.

\section{Histopathology}

Tissue specimens of lung and nasal cavity were fixed in $4 \%$ paraformaldehyde solution for 24 hours. Lung tissues were washed with deionized water and embedded in paraffin. Nasal tissues were also washed with deionized water, decalcified for 3 to 4 weeks and embedded in paraffin. Tissue sections ( $3 \mu \mathrm{m}$ thickness) were stained using a hematoxylin and eosin solution (for qualitative evaluation of the histopathologic change), periodic acid-Schiff solution (for mucus) and Sirius Red staining (for evaluation of eosinophilic infiltration). The number of eosinophils infiltrated into $1 \mathrm{~mm}^{2}$ of pulmonary parenchyma was counted in 20 random high-power fields $(200 \times$ magnification). The number of eosinophils infiltrated into $1 \mathrm{~mm}^{2}$ of lamina propria was counted in 10 high-power fields $(400 \times$ magnification) of the $\mathrm{T} 1$ area (the section was taken immediately caudal to the upper incisor teeth). Two impartial, blinded researchers performed the histopathologic examinations and counted the eosinophils in tissue specimens.

\section{Statistical analysis}

All statistical analyses were conducted with SPSS version 19.0 software (IBM, Armonk, NY). We used the Kruskal-Wallis test and Mann-Whitney $U$-test with Bonferroni adjustment for intergroup comparisons, $p<0.05$ was considered significant.

\section{Results}

\section{Luteolin treatment caused a significant decrease in inflammatory cells in BAL fluid}

After OVA challenge, the number of inflammatory cells such as eosinophils, neutrophils and lymphocytes in BAL fluid was significantly increased in group B (allergic group) compared to group A (nonallergic group, $p<0.01$ ). Mice in group $\mathrm{C}$ (null treatment group with sterile saline) had no significant difference in their number of inflammatory cells in BAL fluid compared to those in group B (all $p>0.05$ ). On the other hand, mice in group $\mathrm{D}$ (treatment group by luteolin) showed a decrease in all inflammatory cells in their BAL fluid compared to group B, although not statistically significant (Fig. 1).

\section{Luteolin treatment induced a significant decrease in Th2 cytokines in lung parenchyma}

After treatment with luteolin, mice in group D showed a modest decrease in IL-4, IL-5 and IL-13 in their lung homogenate tissue compared to groups B and C (Fig. 2).

\section{Luteolin treatment significantly ameliorated the allergic inflammatory lesion of lung and nasal cavity}

Mice in groups B and C had significantly more infiltration of inflammatory cells around bronchioles and in the nasal mucosa compared to those in group A. After luteolin treatment, we could observe a significant decrease in the inflammatory cell infiltration in group D (Fig. 3). The numbers of infiltrated eosinophils around a single bronchiole also significantly decreased after the luteolin treatment (Fig. 4). 

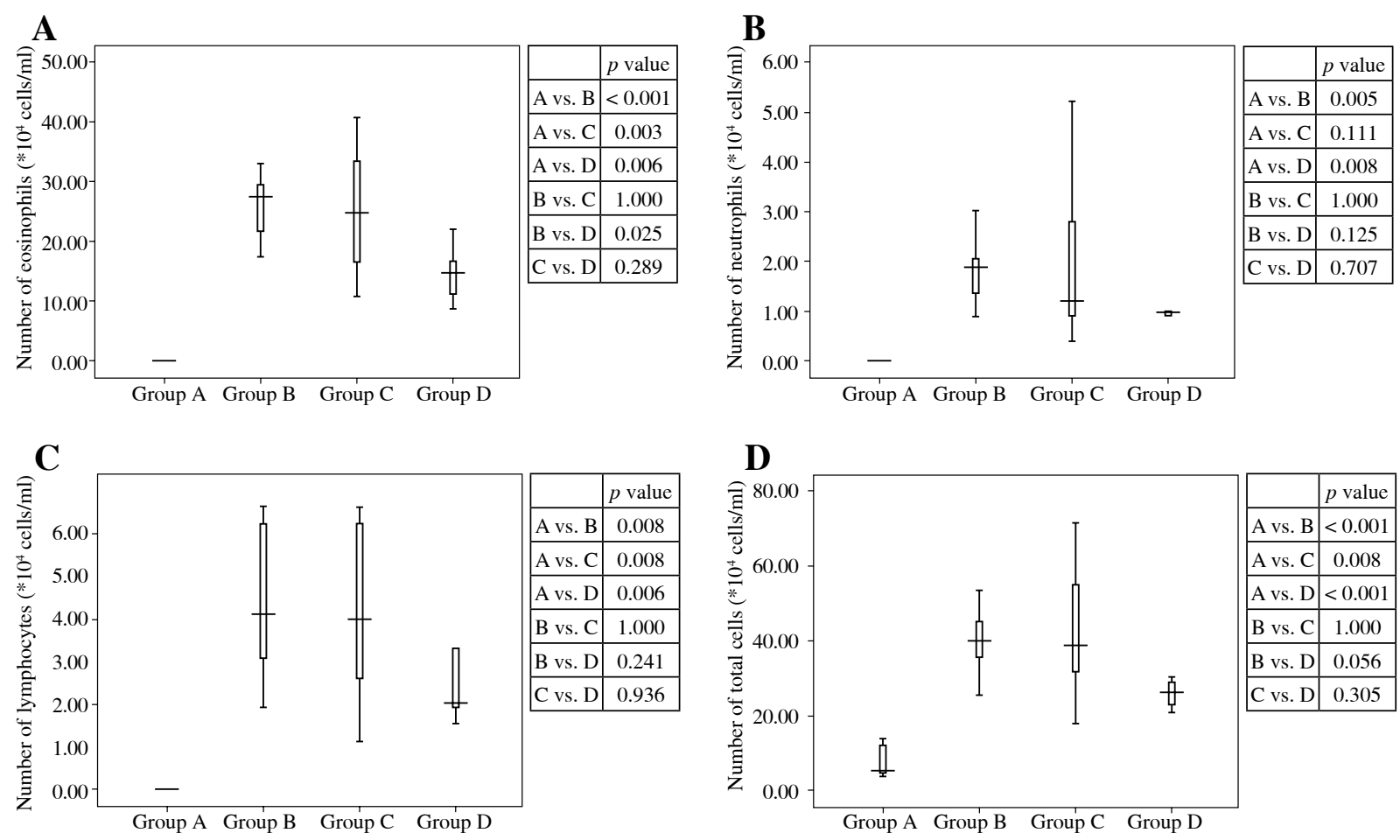

Fig. 1. Number of eosinophils, neutrophils, lymphocytes and total cells in broncho-alveolar lavage fluid. Group A (nonallergic group), Group B (allergic group), Group C (null treatment group) and group D (luteolin treatment group) (Kruskal-Wallis test and Mann-Whitney $\mathrm{U}$ test with Bonferroni adjustment)

A

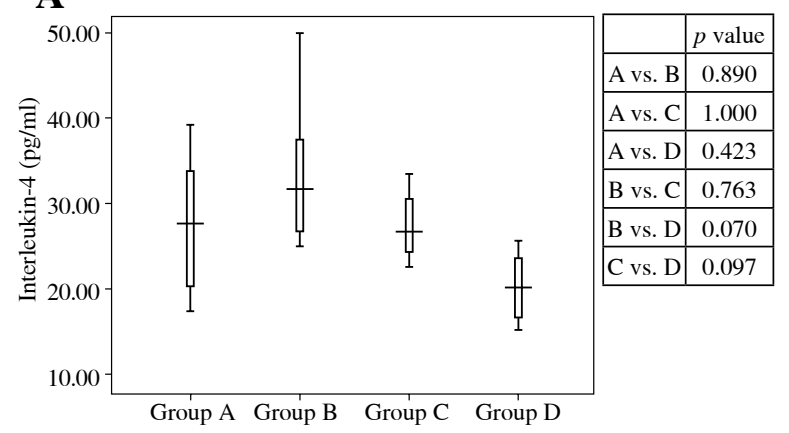

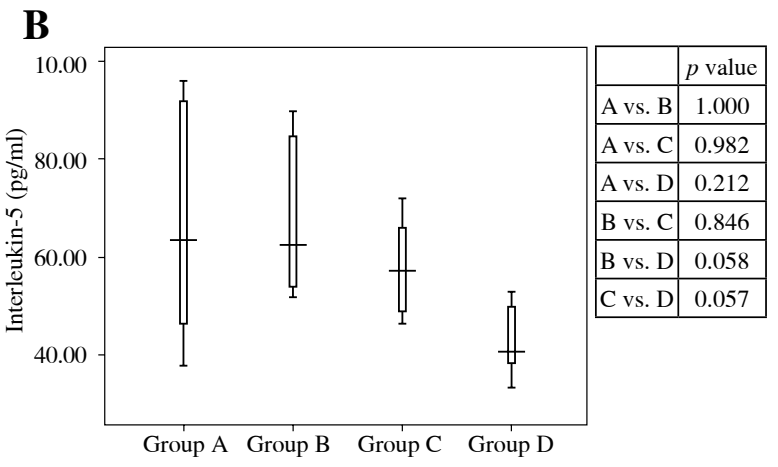

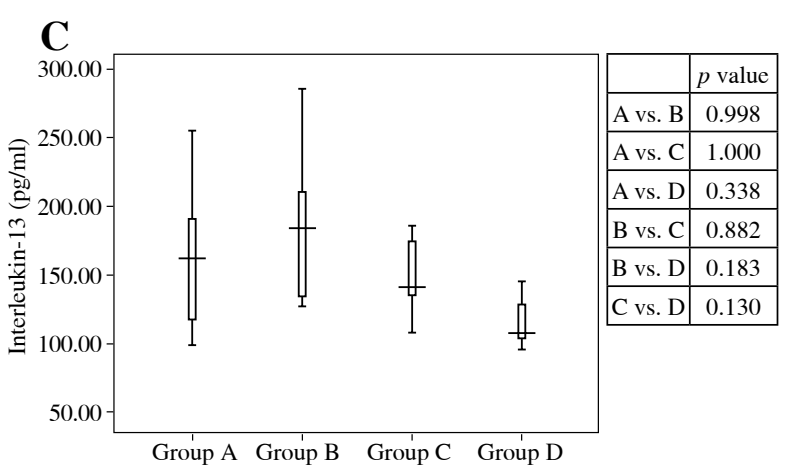

Fig. 2. Titers of IL-4, IL-5 and IL-13 in lung homogenate. Group A (nonallergic group), Group B (allergic group), Group C (null treatment group) and group D (luteolin treatment group) (Kruskal-Wallis test and Mann-Whitney U test with Bonferroni adjustment) 
Lung (magnification 200x)
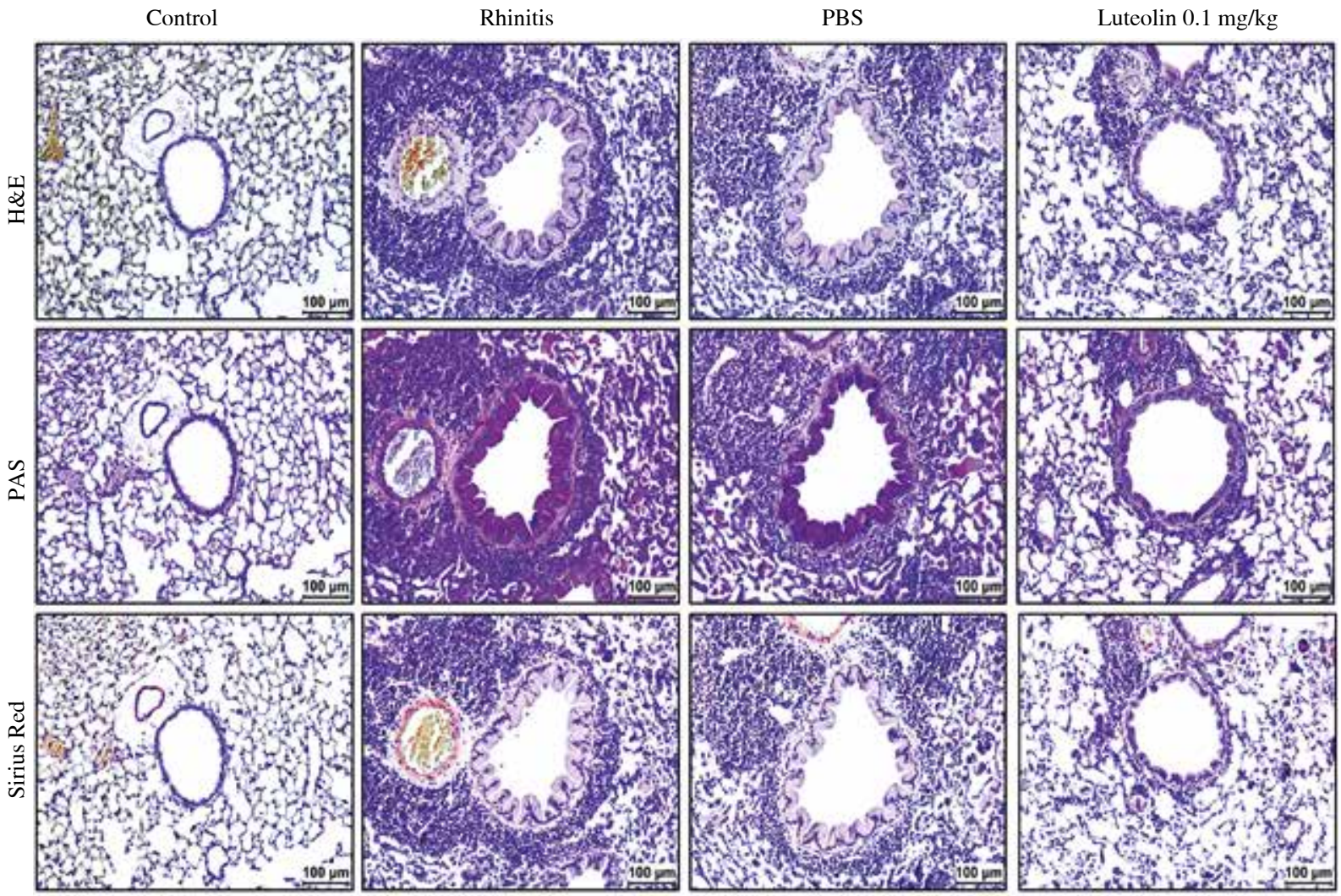

Nose (magnification 200x)
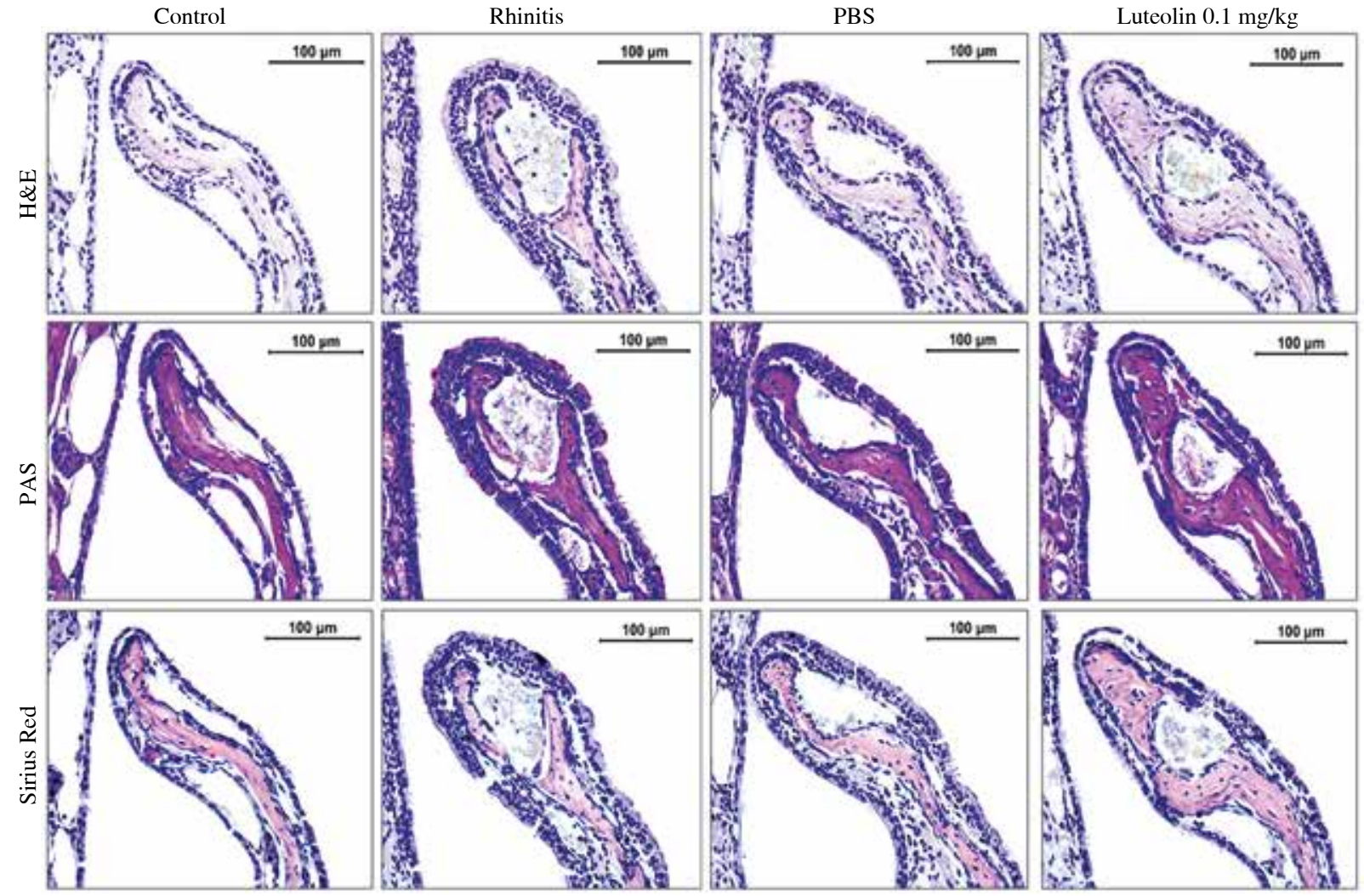

Fig. 3. Histopathologic examination of the lung tissue and nasal cavity 


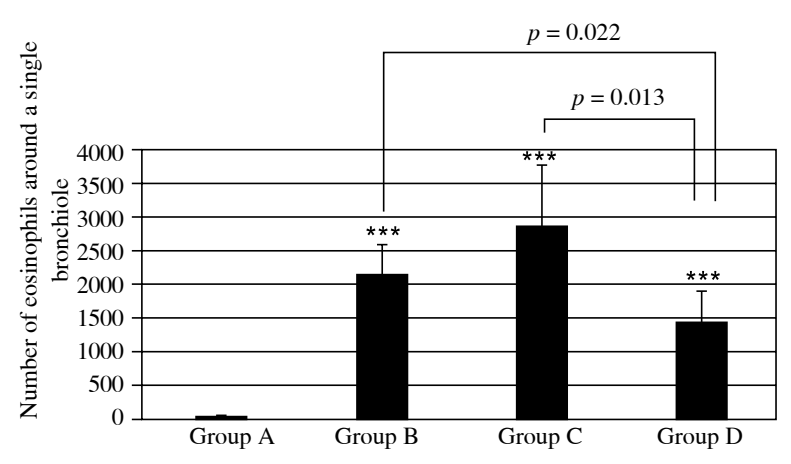

\begin{tabular}{|l|c|}
\hline & $p$ value \\
\hline A vs. $\mathrm{B}$ & $<0.001$ \\
\hline A vs. $\mathrm{C}$ & $<0.001$ \\
\hline A vs. $\mathrm{D}$ & $<0.001$ \\
\hline B vs. $\mathrm{C}$ & 1.000 \\
\hline B vs. $\mathrm{D}$ & 0.022 \\
\hline C vs. $\mathrm{D}$ & 0.013 \\
\hline
\end{tabular}

Fig. 4. The number of infiltrated inflammatory cells in $1 \mathrm{~mm}^{2}$ of lung parenchyma (Kruskal-Wallis test and Mann-Whitney U test with Bonferroni adjustment)

\section{Discussion}

In our study, we could find that the number of inflammatory cells in BAL fluid was significantly decreased after luteolin treatment. In prior studies, some researchers suggested that luteolin significantly ameliorated bronchial hyperresponsiveness in animals with allergic asthma [1, $6]$. However, to the best of our literature review, this is the first study which proved the decreased inflammatory cells in BAL fluid. It is probable that a decrease in eosinophils, neutrophils and lymphocytes could contribute to the clinical improvement of allergic asthma.

After luteolin treatment, we could find that the titers of Th2 cytokines such as IL-4, IL-5 and IL-13 were all significantly decreased in pulmonary parenchyma. In their previous study, Das and researchers suggested that luteolin treatment significantly decreased the titer of IL-5 in BAL fluid. However, the titer of IL-4 in BAL fluid showed no significant change [1]. It is possible that dilution of BAL fluid had an impact on the result. Based on our findings, we could suggest that luteolin treatment could ameliorate Th2 response in individuals with allergic disorders such as allergic asthma and rhinitis. These cytokines play pivotal roles in the initiation and maintenance of allergic cascade [6]. Interleukin 4 could promote Th2 cell differentiation, synthesis of $\operatorname{IgE}$ and up-regulation of receptors for $\operatorname{IgE}$, and mucus secretion [7-9]. Interleukin 5 could increase eosinophilic inflammation and airway infiltration [10]. Interleukin 13 induces B cell differentiation and change of their isotype to IgE [11]. Through the inhibition of these actions of Th2 cytokines, luteolin treatment could exert its therapeutic effects on allergic asthma and rhinitis.

To confirm the actual anti-allergic effect of luteolin, we performed histological examination of the target organ including lung and nasal cavity. In this study, by demonstrating the decrease in inflammatory cell infiltration into the pulmonary parenchyma and nasal mucosa, we can conclude that luteolin treatment had a therapeutic effect on experimental airway inflammation of the upper and lower airway.

One advantage of our study is that we used quite a small amount of luteolin $(0.1 \mathrm{mg} / \mathrm{kg})$. On the other hand, Das and researchers used up to $10.0 \mathrm{mg} / \mathrm{kg}$ of luteolin for evaluating its anti-allergic effect [1]. Another merit of this study is that we demonstrated findings of upper airway (nasal mucosa) and lower airway (lung), adding the proof in accordance with the 'unified airway disease' concept.

The proposed mechanisms of luteolin treatment are known as inhibition of: (a) nuclear transcription factor $\kappa B$ pathway and expression of related genes [12], (b) pro-inflammatory cytokines, including IL-5 [13], (c) release of mediators such as prostaglandins and leukotrienes [12], and (d) expression of TNF- $\alpha$ induced ICAM molecules on endothelial cells [14]. Further study to elucidate the pharmacologic mechanism of luteolin could yield more meaningful data. Also, we need to further confirm our findings in a larger population as our study only included 8 mice in each group.

In conclusion, luteolin treatment had an anti-allergic effect including less infiltration of inflammatory cells and decrease in $\mathrm{Th} 2$ cytokines in a murine model of allergic asthma and rhinitis.

This study was supported by the Inha University Hospital Research Grant.

The authors declare no conflict of interest.

\section{References}

1. Das M, Ram A, Ghosh B (2003): Luteolin alleviates bronchoconstriction and airway hyperreactivity in ovalbumin sensitized mice. Inflamm Res 52: 101-106.

2. Kim YH, Yang TY, Park CS, et al. (2012): Anti-IL-33 antibody has a therapeutic effect in a murine model of allergic rhinitis. Allergy 67: 183-190.

3. Kim YH, Park CS, Lim DH, et al. (2012): Beneficial effect of anti-interleukin-33 on the murine model of allergic inflammation of the lower airway. J Asthma 49: 738-743.

4. Saito H, Matsumoto K, Denburg AE, et al. (2002): Pathogenesis of murine experimental allergic rhinitis: a study of local and systemic consequences of IL-5 deficiency. J Immunol 168: 3017-3023.

5. El Gazzar M, El Mezayen R, Marecki JC, et al. (2006): Anti-inflammatory effect of thymoquinone in a mouse model of allergic lung inflammation. Int Immunopharmacol 6: 1135-1142.

6. Finkelman FD, Urban JF (2001): The other side of the coin: the protective role of the TH2 cytokines. J. Allergy Clin Immunol 107: 772-780. 
7. Seder RA, Paul WE, Davis MM, et al. (1992): The presence of interleukin 4 during in vitro priming determines the lymphokine-producing potential of CD4+ T cells from $\mathrm{T}$ cell receptor transgenic mice. J Exp Med 176: 1091-1098.

8. Pawankar R, Okuda M, Yssel H, et al. (1997): Nasal mast cells in perennial allergic rhinitics exhibit increased expression of the $\mathrm{Fc}$ epsilonRI, CD40L, IL-4, and IL-13, and can induce IgE synthesis in B cells. J Clin Invest 99: 1492-1499.

9. Dabbagh K, Takeyama K, Lee HM, et al. (1999): IL-4 induces mucin gene expression and goblet cell metaplasia in vitro and in vivo. J Immunol 162: 6233-6237.

10. Foster PS1, Hogan SP, Ramsay AJ, et al. (1996): Interleukin 5 deficiency abolishes eosinophilia, airways hyperreactivity, and lung damage in a mouse asthma model. J Exp Med 183: 195-201.

11. Cocks BG, de Waal Malefyt R, Galizzi JP, et al. (1993): IL-13 induces proliferation and differentiation of human $\mathrm{B}$ cells activated by the CD40 ligand. Int Immunol 5: 657-663.

12. Xagorari A, Papapetropoulos A, Mauromatis A, et al. (2001): Luteolin inhibits an endotoxin-stimulated phosphorylation cascade and proinflammatory cytokine production in macrophages. J. Pharmacol Exp Ther 296: 181-187.

13. Park KY, Lee SH, Min BK, et al. (1999): Inhibitory effect of luteolin 4'-O-glucoside from Kummerowia striata and other flavonoids on interleukin-5 bioactivity. Planta Med 65: 457-459.

14. Shimoi K, Saka N, Kaji K, et al. (2000): Metabolic fate of luteolin and its functional activity at focal site. BioFactors Oxf Engl 12: 181-186. 